\title{
The Bond and Cycle Spaces of an Infinite Graph
}

\author{
Karel Casteels \\ Simon Fraser University \\ R. Bruce Richter \\ University of Waterloo
}

\begin{abstract}
Bonnington and Richter defined the cycle space of an infinite graph to consist of the sets of edges of subgraphs having even degree at every vertex. Diestel and Kühn introduced a different cycle space of infinite graphs based on allowing infinite circuits. A more general point of view was taken by Vella and Richter, thereby unifying these cycle spaces. In particular, different compactifications of locally finite graphs yield different topological spaces that have different cycle spaces.

In this work, the Vella-Richter approach is pursued by considering cycle spaces over all fields, not just $\mathbb{Z}_{2}$. In order to understand "orthogonality" relations, it is helpful to consider two different cycle spaces and three different bond spaces. We give an analogue of the "edge tripartition theorem" of Rosenstiehl and Read and show that the cycle spaces of different compactifications of a locally finite graph are related.
\end{abstract}

\section{Introduction}

Diestel and Kühn developed a theory of cycle spaces for the Freudenthal compactification of a locally finite graph [3]. Thinking of the graph as a 1-dimensional cell complex and adding the "ends", the resulting topological space has embeddings of the unit circle, and these are permitted to cover all the edges in two rays (or 1-way infinite paths) having the same origin, but otherwise disjoint, that are in the same end, since the union of these two rays, plus their common end, is a homeomorph of a circle. The cycle space is then taken as the space generated by special, possibly infinite, symmetric differences of the edge sets contained in circles. They prove that the fundamental cycles of an end-faithful spanning tree generate this cycle space. In the later work [4], this is extended to a certain more general topological space $\tilde{G}$ that can be obtained from some slightly more general infinite graph $G$. Vella and Richter [7] show that these results are special cases of a more general theory that includes, for locally finite graphs, the cycle space notion of Bonnington and Richter [1], in which a set of edges of a locally finite graph 
is in this new cycle space if every vertex is incident with an even number of edges in the set. This cycle space is larger than the Diestel-Kühn space for a locally finite graph having more than one end. It is implicit in all of these works that the cycle space is a vector space over the field $\mathbb{Z}_{2}$.

In this work, we do three things. First, and principally, we pursue the Vella-Richter approach to allow any field. Thus, along with other basic results, we show the "directed" fundamental cycles generate the "directed" cycle and bond spaces. This parallels the fact that cycle and bond spaces of finite graphs exist over any field. Over an arbitrary field, we establish various orthogonality relations between cycle spaces and bond spaces. The main new technical tool is Theorem 4.3, which gives a sufficient condition for a subspace $\mathcal{U}$ to satisfy the orthogonality relation $\left(\mathcal{U}^{\perp}\right)^{\perp}=\mathcal{U}$. Secondly, we conjecture a generalization, for any compactification of a locally finite graph, of the Rosenstiehl-Read Edge Tripartition Theorem that says (for $\mathbb{Z}_{2}$ ) every edge of a finite graph is either in a set of edges that is simultaneously in the cycle space and in the bond space, or is the sum of an element of the cycle space with an element of the bond space. We prove an analogue of this result for compactifications of locally finite graphs; this turns out to be a simple application of Theorem 4.3. Finally, in pointing toward future work, we show that if $f: X \rightarrow Y$ is a continuous function that is a bijection on the edges, then the cycle space of $X$ is contained in the cycle space of $Y$. This is especially interesting when $X$ and $Y$ are different compactifications of the same locally finite graph and focuses attention to the problem addressed in [1], which, in a very special case, shows how to find a basis for the quotient of the two cycle spaces.

The topological spaces considered by Vella and Richter are "compact, weakly Hausdorff edge spaces". A space $X$ is weakly Hausdorff if, for any $x, y \in X$, there are open sets $U_{x}$ and $U_{y}$ containing $x$ and $y$, respectively, so that $U_{x} \cap U_{y}$ is finite. An edge space is a pair $(X, E)$ consisting of a topological space $X$ together with a subset $E$ of open singletons in $X$, each having at most two boundary points. Every graph $G=(V, E)$ can be converted into a weakly Hausdorff edge space $(V \cup E, E)$ by defining the basic open sets as follows: for each edge $e,\{e\}$ is open, while for each vertex $v$, the set consisting of $v$ and its incident edges is open. Other variants include allowing additional points to make the space compact. Specific examples are the 1-point compactification and the Freudenthal compactification of a locally-finite graph. In [1], the closure of an embedding of a locally finite graph into the sphere is considered. See [7] for details. 
The study of infinite cycles in graphs has received substantial attention lately and many theorems for finite graphs have been successfully generalized to this setting. Diestel surveys many of these in [2].

\section{Cycles and Bonds}

In this short section, we review a few results from [7] that are central to the theory of cycle and bond spaces. An edge space $(X, E)$ is an edge cycle if: (i) $X$ is connected; (ii) for each $e \in E, X-e$ is connected; and (iii) for distinct $e, f \in E, X-\{e, f\}$ is not connected. A cycle is the set of edges of an edge cycle. A spanning tree of $(X, E)$ is a connected subspace $T$ of $X$ containing $X \backslash E$ such that, for all $e \in T \cap E, T-e$ is not connected. To define a bond, let $(A, B)$ be a partition of $X \backslash E$ into closed sets. The bond $\delta(A)=\delta(B)$ is the set of edges $e$ having one boundary point in $A$ and other in $B$.

The following facts are [7, Cor. 5 andThm. 12], respectively.

Theorem 2.1 Every connected, compact, weakly Hausdorff edge space has a spanning tree.

Theorem 2.2 Every bond in a compact, weakly Hausdorff edge space is finite.

Let $\mathrm{T}$ be a spanning tree in a connected, compact, weakly Hausdorff edge space $(X, E)$ and let $e \in E \backslash T$. It is a consequence of [7, Thm. 3] that $T+e$ contains an edge cycle containing $e$ (use $e$ together with the edge set of a minimal, closed, connected set in $T$ containing the ends of $e$ ) - the corresponding cycle is the fundamental cycle $C_{T}(e)$. For $e \in T \cap E, T-e$ has precisely two components $A$ and $B$. Both $A \backslash E$ and $B \backslash E$ are closed, so $\delta(A)$ is a bond - this is the fundamental bond $B_{T}(e)$. The following is $[7$, Lemma 16].

Lemma 2.3 Let $T$ be a spanning tree in a compact, weakly Hausdorff edge space $(X, E)$, let $e \in E \backslash T$ and let $f \in T \cap E$. Then $e \in B_{T}(f)$ if and only if $f \in C_{T}(e)$.

A central property that makes everything work follows immediately from Theorem 2.2 and Lemma 2.3. A set $S$ of subsets of a set $A$ is thin if every element of $A$ is in only finitely many sets in $S$.

Theorem 2.4 Let $T$ be a spanning tree of a compact, weakly Hausdorff edge space $(X, E)$. The set $\left\{C_{T}(e) \mid e \in E \backslash T\right\}$ of fundamental cycles is thin. 


\section{Cycle and Bond Spaces}

In this section we introduce the cycle and bond spaces of a compact, weakly Hausdorff edge space $(X, E)$, over any field $\mathbb{F}$. We start by choosing an arbitrary, but fixed, orientation for each edge in $E$. The space $\mathbb{F}^{E}$ consists of all formal linear combinations $\sum_{e \in E} \alpha_{e} e$, with all $\alpha_{e}$ being in $\mathbb{F}$. It may at times be convenient to view $\mathbb{F}^{E}$ as the set of vectors $\left(\alpha_{e}\right)$ indexed by $E$, or as the set of functions $f: E \rightarrow \mathbb{F}$.

It is a triviality that $\mathbb{F}^{E}$ is a vector space (with addition and scalar multiplication defined component-wise), but this is not so relevant for us. The support $\xi\left(\sum_{e \in E} \alpha_{e} e\right)$ of an element $\sum_{e \in E} \alpha_{e} e$ of $\mathbb{F}^{E}$ is the set $\left\{e \in E \mid \alpha_{e} \neq 0\right\}$. We extend the notion of "thinness" to subsets of $\mathbb{F}^{E}$ as follows: a subset $S$ of $\mathbb{F}^{E}$ is thin if, for each $e \in E$, the set $\{s \in S \mid e \in \xi(s)\}$ is finite.

For a subset $S$ of $\mathbb{F}^{E}$, we can identify three subsets of $\mathbb{F}^{E}$ "generated by $S$ ". First, the weak span $\mathcal{W}(S)$ is the set of all finite linear combinations of the elements of $S$. Next, the algebraic span $\mathcal{A}(S)$ is the set of all (possibly infinite) linear combinations of all thin subsets $S^{\prime}$ of $S$. Note that such sums make sense, since $\sum_{s \in S^{\prime}} \alpha_{s} s$ may be defined to be $\sum_{e \in E}\left(\sum_{s \in S^{\prime}} \alpha_{s} s_{e}\right) e$, where $s=\sum_{e \in E} s_{e} e$. The set $S$ is algebraically closed if $\mathcal{A}(S)=S$. The strong span $\mathcal{S}(S)$ is the intersection of all algebraically closed sets $W \subseteq \mathbb{F}^{E}$ such that $S \subseteq W$.

An example may be helpful. Let $E=\left\{e_{1}, e_{2}, \ldots\right\}$, let $\mathbb{F}=\mathbb{Z}_{2}$, let $D$ be the set of all pairs from $E$, and let $D_{1}=\left\{\left\{e_{1}, e_{i}\right\} \mid i \geq 2\right\}$. Then $D_{1}$ is not thin - the only thin subsets of $D_{1}$ are the finite subsets. The sets $\mathcal{W}(D)$ and $\mathcal{A}\left(D_{1}\right)$ consist of all the finite subsets of $\left\{e_{1}, e_{2}, \ldots\right\}$ having an even number of edges. But $\mathcal{S}(D)=\mathcal{S}\left(D_{1}\right)$ consists of all subsets of $\left\{e_{1}, e_{2}, \ldots\right\}$ and in this case $\mathcal{S}\left(D_{1}\right)=\mathcal{A}\left(\mathcal{A}\left(D_{1}\right)\right)=\mathcal{A}(D)$, while, for any set $S, \mathcal{W}(\mathcal{W}(S))=\mathcal{W}(S)$.

For each cycle $C$ of $(X, E)$, there is an edge cycle $\bar{C}$ whose edge set is $C$. As shown in [8], Theorem 5.1.6 (see also, [7], Lemma 7 and Corollary 9 ), there is a natural cyclic orientation of the edges, which obviously extends to include their incident vertices, so that if $e$ is any edge of $C$ with ends $u$ and $v, u, e, v$ are consecutive (either in that or its inverse order). In this cyclic orientation, if the edges $e_{1}, e_{2}, e_{3}, e_{4}$ occur in this order, then $e_{2}$ and its incident vertices are in one component of $\bar{C} \backslash\left\{e_{1}, e_{3}\right\}$ and $e_{4}$ and its incident vertices are in the other. There are two such orientations. In order to proceed, we arbitrarily fix $D$ to be one of these orientations. Each edge $e$ of $C$ has its original orientation either in agreement with $D$ - we say $e$ is positive in this case - or it does not - in which case $e$ is negative. We set $D^{+}$ 
and $D^{-}$to be the sets of positive edges and negative edges, respectively, in $C$. Then $D$ gives rise to the following element of $\mathbb{F}^{E}$ :

$$
z_{D}=\left(\sum_{e \in D^{+}} e\right)-\left(\sum_{e \in D^{-}} e\right) .
$$

Notice that if $D^{\prime}$ is the other orientation of $C$, then $z_{D^{\prime}}=-z_{D}$.

In particular, if $C$ is the fundamental cycle $C_{T}(e)$, then we also use the label $C_{T}(e)$ for $z_{D}$, where $D$ is the orientation of $C$ chosen to have $e$ positive. This abuse of notation should not cause any confusion, since we will no longer have use for the unoriented fundamental cycle.

The cycle space $\mathcal{Z}_{t}$ of the compact, weakly Hausdorff edge space $(X, E)$ is the strong span of the set of $z_{D}$. The finite cycle space $\mathcal{Z}_{f}$ is the set of all elements $\sum \alpha_{e} e$ of $\mathcal{Z}_{t}$ having finite support.

As with finite graphs, there is a dual way to think about $\mathcal{Z}_{t}$. For a bond $\delta(A)$, let $\delta^{+}(A)$ denote the set of edges $e \in \delta(A)$ whose head is in $A$ (is pointed in to $A$ ) and let $\delta^{-}(A)$ denote the set of edges $e \in \delta(A)$ whose tail is in $A$ (is pointed out from $A$ ). If $(A, B)$ is a partition of $X \backslash E$ into two closed sets, then $\delta^{+}(A)=\delta^{-}(B)$. We again abuse notation and use the symbol $\delta(A)$ for $\sum_{e \in \delta^{-}(A)} e-\sum_{e \in \delta^{+}(A)} e$. We will also call this a bond - we will no longer have much need for the unoriented object, so again no confusion should arise. The finite bond space $\mathcal{B}_{f}$ is the weak span of the set of bonds $\delta(A)$.

For two vectors $a=\sum \alpha_{e} e$ and $b=\sum \beta_{e} e$ in $\mathbb{F}^{E}$, we define $a \circ b$ to be the sum $\sum_{e \in E} \alpha_{e} \beta_{e}$. We note that this sum is not always defined, but, for the moment, we are concerned only with the case that $a$ has finite support i.e., only finitely many $\alpha_{e}$ are non-zero. We note the following easy fact.

Lemma 3.1 Let $B$ be a thin subset of $\mathbb{F}^{E}$ and let $a \in \mathbb{F}^{E}$ have finite support. Then $a \circ \sum_{b \in B} b=\sum_{b \in B} a \circ b$.

The set $\mathcal{B}_{f}^{\perp}$ consists of all vectors $a \in \mathbb{F}^{E}$ such that, for all $b \in \mathcal{B}_{f}, a \circ b=0$. Lemma 3.1 shows that $\mathcal{B}_{f}^{\perp}$ is closed under thin sums.

A set $S$ is an algebraic basis for a set $T$ if it algebraically spans, i.e., $T=\mathcal{A}(S)$, and is algebraically independent, i.e., whenever $S^{\prime}$ is a thin subset of $S$ and $\sum_{s \in S^{\prime}} \alpha_{s} s=0$, then all the $\alpha_{s}$ are 0 . Note that any linearly spanning set algebraically spans, but not always conversely, and that any algebraically independent set is linearly independent, but not always conversely. We have the following version of a standard result for finite graphs; this is the first of our orthogonality relations. 
Theorem 3.2 Let $(X, E)$ be a compact, connected, weakly Hausdorff edge space. Then $\mathcal{Z}_{t}=B_{f}^{\perp}$. Furthermore, the set of fundamental cycles for any spanning tree form an algebraic basis for $\mathcal{Z}_{t}$.

Proof: We first show that $B_{f}^{\perp}$ is algebraically closed. Let $a=\sum_{i=1}^{k} \alpha_{i} \delta\left(A_{i}\right)$ be any element of $B_{f}$. Let $S \subseteq B_{f}^{\perp}$ be thin. Lemma 3.1 implies $a \circ \sum_{s \in S} s=$ $\sum_{s \in S} a \circ s$. Each term in the latter sum is 0 , as required.

To show $Z_{t} \subseteq B_{f}^{\perp}$, we extract the following observation.

Lemma 3.3 Let $D$ be an orientation of a cycle $C$ and let $b \in B_{f}$. Then $z_{D} \circ b=0$.

Proof: By Lemma 3.1, we may assume that $b=\delta(A)$, for some partition $(A, B)$ of $X \backslash E$ into closed sets. Then $C \backslash(\delta(A) \cap C)$ has $|\delta(A) \cap C|$ components, which are cyclically joined by the edges of $\delta(A) \cap C$. Let $e_{1}, e_{2}, \ldots, e_{k}$ denote the cyclic order of the edges of $\delta(A) \cap C$. Then $k$ is even. The crucial observation to show that $e_{i}$ and $e_{i+1}$ contribute cancelling values is the following: if $e_{i}$ and $e_{i+1}$ are both in $D^{+}$or both in $D^{-}$, then one is in $\delta^{+}(A)$ and the other is in $\delta^{-}(A)$, while if they are in different ones of $D^{+}$and $D^{-}$, then either both are in $\delta^{+}(A)$ or both are in $\delta^{-}(A)$. The various possibilities are now easily dismissed.

This lemma shows that each $z_{D}$ is in $B_{f}^{\perp}$. Thus, $B_{f}^{\perp}$ is an algebraically closed set containing all the $z_{D}$, i.e., $Z_{t} \subseteq B_{f}^{\perp}$.

For the reverse inclusion, let $c=\sum \alpha_{e} e \in B_{f}^{\perp}$. Choose any spanning tree $T$ of $(X, E)$, and let $z=c-\sum_{e \notin T} \alpha_{e} C_{T}(e)$. Since the fundamental cycles are thin, $\sum_{e \notin T} \alpha_{e} C_{T}(e) \in B_{f}^{\perp}$ from the first part of the proof and Lemma 3.1. Thus, $z \in B_{f}^{\perp}$. Furthermore, the support of $z$ is contained in $T$. Now let $e \in T$. Recall that $\xi\left(B_{T}(e)\right) \cap T=\{e\}$. Since $z \circ B_{T}(e)=0$, it follows that $e \notin \xi(z)$. Thus, $z=0$, i.e., $c \in \mathcal{Z}_{t}$, as required. Furthermore, this proves that the fundamental cycles algebraically span the cycle space.

It remains to show algebraic independence, so suppose $\sum_{e \notin T} \alpha_{e} C_{T}(e)=0$. For $e \notin T, C_{T}(e)$ is the only fundamental cycle whose support contains $e$. Thus, when we write $\sum_{e \notin T} \alpha_{e} C_{T}(e)$ in the form $\sum_{f \in E} \beta_{f} f$, we must have, for each $e \notin T, \beta_{e}=\alpha_{e}$. Thus, every $\alpha_{e}$ is 0 , as required.

As a particular instance, for a locally finite graph $G$, consider the elements $\sum \alpha_{e} e$ of $\mathbb{F}^{E}$ having the property that, for every vertex $v$ of $G, \sum_{e \in \delta^{+}(v)} \alpha_{e}=$ 
$\sum_{e \in \delta^{-}(v)} \alpha_{e}$. (This is the Bonnington-Richter cycle space, but now for a general field.) This is precisely $B_{f}^{\perp}$, and so $\mathcal{Z}_{t}$, for the Alexandroff (or 1point) compactification of $G$. This is a larger space than the cycle space of the Freudenthal compactification of a locally-finite $G$ having more than one end.

\section{More on orthogonality}

In the previous section, we showed that $B_{f}^{\perp}=\mathcal{Z}_{t}$. In general $\mathcal{Z}_{t}^{\perp}$ is not $B_{f}$. It is the purpose of this section to determine $\mathcal{Z}_{t}^{\perp}$. While we are at it, let $\Delta$ denote the set of all the bonds $\delta(A)$ and let $B_{t}=\mathcal{A}(\Delta)$. For a locally finite graph, we show that $B_{t}^{\perp}=\mathcal{Z}_{f}$ and $\mathcal{Z}_{f}^{\perp}=B_{t}$; it is interesting to note that $\mathcal{Z}_{f}$ and $B_{t}$ depend only on the graph and not on the compactification.

In order to determine $\mathcal{Z}_{t}^{\perp}$, it is necessary to reconsider the meaning of $a \circ b$. By definition, this should be $\sum_{e \in E} \alpha_{e} \beta_{e}$, where $a=\sum_{e \in E} \alpha_{e} e$ and $b=\sum_{e \in E} \beta_{e} e$. Which sums $\sum_{e \in E} \gamma_{e}$ are to be permitted? In the proofs that follow, we will need to be able to add such sums, to rearrange the terms without changing the value of the sum, along with other natural operations. For a finite field, the only possibility that makes sense in this context is finite sums. However, for an infinite field, the absolutely convergent sums also make sense. Thus, for these fields, we shall have a choice: allow only finite sums or allow absolutely convergent sums. Since both of these options make sense, we let $\mathcal{C}$ denote the set of allowed sums. We shall use without comment the properties we require of $\mathcal{C}$; the reader may note along the way that both the set of finite sums and the set of absolutely convergent sums satisfy all the properties we use.

The function $\circ$ is then dependent on $\mathcal{C}$ and $a \circ b$ is only defined if the sum $\sum \alpha_{e} \beta_{e}$ is in $\mathcal{C}$. For a subset $S$ of $\mathbb{F}^{E}$, we define $S^{\perp}=\left\{a \in \mathbb{F}^{E} \mid \forall s \in\right.$ $S, a \circ s \in \mathcal{C}, a \circ s=0\}$. It is a triviality to show that $S \subseteq\left(S^{\perp}\right)^{\perp}$ and that if $U \subseteq V$, then $V^{\perp} \subseteq U^{\perp}$. It is in general not true that $\left(S^{\perp}\right)^{\perp}=S$; we will see that, at least in some cases, $B_{f} \neq\left(B_{f}^{\perp}\right)^{\perp}$.

A simple example will help to illustrate. Let $G$ be a 2-way infinite path, every edge oriented from one end of $G$ towards the other end and let $X$ be the 1-point compactification of $G$. If $\mathbb{F}$ is the real numbers, then $\mathcal{Z}_{t}$ consists of all the real multiples of the vector $z$ of all 1's. Fix some vertex $v$ of $G$ to be the "midpoint" of $G$. Let $a$ be a vector in $\mathbb{F}^{E(G)}$ obtained by putting $1 /\left(2^{i}\right)$ and $-1 /\left(2^{i}\right)$ on the edges at distance $i$ from $v$. The entries in $a$ make an 
absolutely convergent series, and $a \circ z=0$, so if $\mathcal{C}$ consists of the absolutely convergent sums, then $a \in \mathcal{Z}^{\perp}$. However, if $\mathcal{C}$ consists only of the finite sums, then $a \notin \mathcal{Z}^{\perp}$, since $a \circ z \notin \mathcal{C}$.

The following is easy and well-known.

Lemma 4.1 Let $E$ be any set, let $\mathbb{F}$ any field, and let $A$ and $B$ be subsets of $\mathbb{F}^{E}$. Then $(A+B)^{\perp}=A^{\perp} \cap B^{\perp}$.

We shall see two sufficient conditions to ensure that $\left(A^{\perp}\right)^{\perp}=A$. The first is quite simple and general, while the second relates specifically to algebraic generation.

Lemma 4.2 Let $A$ be a subset of $\mathbb{F}^{E}$. Then $A=\left(A^{\perp}\right)^{\perp}$ if and only if there is a $B$ so that $A=B^{\perp}$.

Proof: This is a special case of a general fact. Suppose $f$ is a function mapping subsets of $\mathbb{F}^{E}$ to subsets of $\mathbb{F}^{E}$ satisfying: (i) for all $A, A \subseteq f(f(A))$; and (ii) for all $A \subseteq B, f(B) \subseteq f(A)$. Then, for every $A, f(A)=f(f(f(A)))$. To see this, note by (i) $f(A) \subseteq f(f(f(A)))$. Also by (i), $A \subseteq f(f(A))$, so by (ii) $f(f(f(A))) \subseteq f(A)$. To finish the proof of the lemma, observe that if $A=f(B)$, then $f(f(A))=f(f(f(B)))=f(B)=A$. Conversely, If $A=f(f(A))$, then, for $B=f(A), A=f(B)$.

This simple lemma and Theorem 3.2 already show that $\left(\mathcal{Z}_{t}^{\perp}\right)^{\perp}=\mathcal{Z}_{t}$. The next result will combine with Theorem 3.2 to do this in a different way. However, the next result will be used later to get our analogue of the edge tripartition theorem.

Theorem 4.3 Let $\mathcal{U}$ be a subspace of $\mathbb{F}^{E}$. If $\mathcal{U}$ is algebraically generated by a thin set, then $\left(\mathcal{U}^{\perp}\right)^{\perp}=\mathcal{U}$.

This is a simple consequence of the following result that generalizes Fredholm's Theorem of the Alternative for finite systems of linear equations to systems of equations in which each equation has only finitely many non-zero terms. Our thanks to Christian Delhommé for the argument, which is much simpler than our original.

Lemma 4.4 Let $A x=b$ be a system of equations in which every equation has only finitely many non-zero coefficients. If every finite set of equations from $A x=b$ has a solution, then $A x=b$ has a solution. 
Proof: Given two systems of equations $S_{1}: A_{1} x=b_{1}$ and $S_{2}: A_{2} x=$ $b_{2}$, we say $S_{1} \leq S_{2}$ if every equation in $S_{1}$ is an equation in $S_{2}$. (The exact same equation should not be repeated.) If $\left\{S_{i}\right\}$ is a chain of systems of equations, every one of which has the property that every finite set of equations has a solution, then $\cup_{i} S_{i}$ is a system of equations with the same property. Therefore, Zorn's Lemma implies there is a maximal system $A^{\prime} x=$ $b^{\prime}$ of equations, containing $A x=b$, so that every finite subsystem of $A^{\prime} x=b^{\prime}$ has a solution.

Suppose $x_{\tau}$ is one of the variables and let $c$ be an element of the field. If $x_{\tau}=c$ is not one of the equations of $A^{\prime} x=b^{\prime}$, then add it to the system. Since this new system properly contains $A^{\prime} x=b^{\prime}$, it must be that some finite subsystem $A_{F}^{\prime} x=b_{F}^{\prime}$ of $A^{\prime} x=b$ has a solution, but for no solution is $x_{\tau}=c$.

We claim that this implies that there is a value $a$ in the field so that, for any solution $x$ to $A_{F}^{\prime} x=b_{F}^{\prime}, x_{\tau}=a$. For if this not the case, let $A_{F}^{\prime \prime} x=b_{F}^{\prime \prime}$ be the reduced row echelon form of $A_{F}^{\prime} x=b_{F}^{\prime}$. There are only finitely many variables that correspond to leading 1 's (i.e., are dependent) in $A_{F}^{\prime \prime} x=b_{F}^{\prime \prime}$; the remainder are independent. If $x_{\tau}$ is independent, then setting all other independent variables to 0 and $x_{\tau}$ to $c$ yields a solution, which is a contradiction. Thus, $x_{\tau}$ must be dependent. The corresponding equation in $A_{F}^{\prime \prime} x=b_{F}^{\prime \prime}$ must be $x_{\tau}=a$, with no independent variables occurring with non-zero coefficient, since otherwise we may again find a solution with $x_{\tau}=c$.

The conclusion is that, for every $\tau$, the value of $x_{\tau}$ is determined by some finite set of equations in $A^{\prime} x=b^{\prime}$. Using these values, in particular, yields a solution to $A^{\prime} x=b^{\prime}$, since no two finite sets of equations can determine distinct values for any $x_{\tau}$.

Our second orthogonal pair is given in the next result. A compactification of a locally finite graph $G$ is a topological space $X \supseteq G$ for which there is a continuous, surjective map $f: \mathbf{F}(G) \rightarrow X$ from the Freudenthal compactification $\mathbf{F}(G)$ of $G$ to $X$ that is the identity on $G$. Lemma 19.8 in [9] shows this implies $f(\mathbf{F}(G) \backslash G)=(X \backslash G)$. Roughly, a compactification of $G$ is obtained from $\mathbf{F}(G)$ by identifying some of the ends. (One could allow more general compactifications than the Freudenthal compactification here. If $R$ and $R^{\prime}$ are rays in the same end of $G$, then the edges occurring once in the 2-way infinite walk $R^{-1} R^{\prime}$ make a set that is the edge-disjoint union of finite cycles of $G$ and so is in every cycle space that allows sums of thin families and contains all finite cycles. However, it is not clear that 
this implies that the cycle space of the Freudenthal compactification is the same as that of, say, the Stone-Cech compactification. Our interest in the context of graphs has principally been devoted to images of $\mathbf{F}(G)$ because the Stone-Cech compactification is not concretely understood. In particular, the Stone-Čech compactification of the positive integers does not yet have an alternative description.)

Theorem 4.5 Let $X$ be a compactification of a connected locally finite graph. Then $B_{t}^{\perp}=Z_{f}$ and $Z_{f}^{\perp}=B_{t}$. Furthermore, the set of vertex bonds $\delta(v)$, less any one, is an algebraic basis for $B_{t}$.

Proof: For each vertex $u$ of $G, \delta(\{u\}) \in B_{f}$. If $z \in Z_{t}$, then $\delta(\{u\}) \circ z=0$ by Theorem 3.2. In particular, $\xi(z)$ cannot have just one edge incident with $u$, so, for $z \in Z_{f}, \xi(z)$ induces a finite subgraph in which no vertex has degree 1. An easy induction on $|\xi(z)|$ now shows that every element of $Z_{f}$ is the finite sum of finite cycles of $G$. Now Lemmas 3.1 and 3.3 show that any element of $Z_{f}$ is orthogonal to any element of $B_{t}$. Thus, $Z_{f} \subseteq B_{t}^{\perp}$ and $B_{t} \subseteq Z_{f}^{\perp}$.

Let $z=\sum \alpha_{e} e \in B_{t}^{\perp}$. Since $B_{f} \subseteq B_{t}$, we have $B_{t}^{\perp} \subseteq B_{f}^{\perp}=Z_{t}$, so it suffices to show $z$ has finite support. Otherwise, there is an infinite set $U$ of pairwise non-adjacent vertices each incident with an edge in the support of $z$. For each $u \in U$, let $e_{u}$ be an edge incident with $u$ such that $\alpha_{e_{u}} \neq 0$. Set $b=\sum_{u \in U} \alpha_{e_{u}}^{-1} \delta(u)$. Clearly $b \in B_{t}$. But now $z \circ b$ is a sum with infinitely many 1's or -1 's, which is impossible, contradicting the assumption that $z \in B_{t}^{\perp}$. Thus, $B_{t}^{\perp} \subseteq Z_{f}$, so $B_{t}^{\perp}=Z_{f}$.

Now suppose $b=\sum \beta_{e} e \in B_{t}$. We shall determine $b^{\prime}=\sum_{v \in V(G)} \alpha_{v} \delta(v)$ so that $b^{\prime}=b$. This shows that $B_{t}$ is algebraically generated by the thin family $\{\delta(v) \mid v \in V(G)\}$, so Theorem 4.3 implies $\left(B_{t}^{\perp}\right)^{\perp}=B_{t}$, i.e., $Z_{f}^{\perp}=B_{t}$.

Let $v_{0}$ be an arbitrary vertex of $G$ and let $T$ be a breadth-first search spanning tree of $G$ rooted at $v_{0}$ - this is independent of $X$, and $T$ need not be a spanning tree of $X$. Set $\alpha_{v_{0}}=0$. Let $v$ be any other vertex of $G$ and let $w$ be the unique neighbour of $v$ closer to $v_{0}$ in $T$. If the edge $v w$ is oriented from $w$ to $v$, then set $\sigma_{v w}=1$, while if $v w$ is oriented from $v$ to $w$, then set $\sigma_{v w}=-1$. Set $\alpha_{v}=\alpha_{w}+\sigma_{v w} \beta_{v w}$. Suppose that $v w$ is an edge of $T$ with $v$ further from $v_{0}$ than $w$ is. If $v w$ is oriented from $v$ to $w$, then $\alpha_{w}-\alpha_{v}=-\sigma_{v w} \beta_{v w}=\beta_{v w}$, while if $v w$ is oriented from $w$ to $v$, then $\alpha_{v}-\alpha_{w}=\sigma_{v w} \beta_{v w}=\beta_{v w}$.

Now consider an edge $v w \in E(G) \backslash E(T)$. There is an orientation $D=$ $\left(w_{0}, e_{0}, w_{1}, e_{1}, \ldots, w_{r}, e_{r}, w_{0}\right)$ of the unique cycle in $T+v w$, with $w=w_{0}$ and 
$v=w_{r}$, so that the orientation of $v w$ is from $v$ to $w$. Then $z_{D} \in \mathcal{Z}_{f}=B_{t}^{\perp}$, so $b \circ z_{D}=0$. Now, if $z_{D}=\sum_{i=0}^{r} \gamma_{i} e_{i}$, then $\sum_{j=0}^{r} \gamma_{i} \beta_{e_{i}}=0$. Now $\gamma_{i}=1$ if $e_{i}$ is oriented from $w_{i}$ to $w_{i+1}$ (indices being read modulo $r+1$ ) and $\gamma_{i}=-1$ otherwise. Thus, for $0 \leq i<r, \gamma_{i} \beta_{e_{i}}=\alpha_{w_{i+1}}-\alpha_{w_{i}}$. So $\sum_{i=0}^{r-1} \gamma_{i} \beta_{e_{i}}=$ $\alpha_{w_{r}}-\alpha_{w_{0}}$. Since $\gamma_{r}=1$, it follows that $\beta_{e_{r}}=\alpha_{w_{0}}-\alpha_{w_{r}}=\alpha_{w}-\alpha_{v}$. Therefore, $b=\sum_{v \in V(G)} \alpha_{v} \delta(v)$, as claimed.

To show that all but one of the vertex bonds make an algebraic basis is easy: the preceding shows $B_{t}$ is the span of the vertex bonds other than $\delta\left(v_{0}\right)$. On the other hand, suppose $a=\sum_{v \in V(G)} \alpha_{v} \delta(v)$. Since $a=\sum_{e \in E}\left(\alpha_{h(e)}-\right.$ $\left.\alpha_{t(e)}\right) e$, where $h(e)$ and $t(e)$ are the head and tail of $e$, respectively, we see that if $a=0$, then $\alpha_{h(e)}=\alpha_{t(e)}$, for every $e$. Since $G$ is connected, all the $\alpha_{v}$ are the same, which proves the claim.

It would be interesting to determine whether Theorem 4.5 holds for general compact, weakly Hausdorff edge spaces. The remainder of this section is devoted to showing, for edge spaces, that $\mathcal{Z}_{t}^{\perp}$ is the space $B_{w}$, which we introduce next.

Let $(X, E)$ be a compact, weakly Hausdorff edge space, let $\mathbb{F}$ be a field, let $\mathcal{C}$ be the set of sums allowed, and let $T$ be a spanning tree of $(X, E)$. The set $B_{w}$ consists of those elements $b$ of $\mathbb{F}^{E}$ that may be expressed in the form $\sum_{e \in E^{\prime}} \alpha_{e} B_{T}(e)$, where $E^{\prime} \subseteq E \cap T$ is such that $\bigcup_{e \in E^{\prime}}\left[\xi\left(B_{T}(e)\right) \backslash\{e\}\right]$ is finite, and, for $F=\left\{e \in E^{\prime} \mid \xi\left(B_{T}(e)\right) \neq\{e\}\right\}, \sum_{e \in F} \alpha_{e} \in \mathcal{C}$. Note that, in the case $\mathcal{C}$ is not just the finite sums, an element of $B_{w}$ is not necessarily the sum of a thin family of $B_{T}(e)$. However, for $f \in E \backslash T$, the coefficient of $f$ in $\sum_{e \in E^{\prime}} \alpha_{e} B_{T}(e)$ is $\sum_{e \in E^{\prime}} \alpha_{e} \mu(e, f)$, where $\mu(e, f)$ is 0 if $f \notin B_{T}(e), 1$ if the coefficients of $e$ and $f$ in $B_{T}(e)$ have the same sign, and -1 otherwise. The sign of $\mu(e, f)$ does not affect whether $\sum_{e \in E^{\prime}, f \in B_{T}(e)} \alpha_{e} \mu(e, f)$ is in $\mathcal{C}$. Since $\sum_{e \in F} \alpha_{e}$ is in $\mathcal{C}$, so is $\sum_{e \in E^{\prime}} \alpha_{e} \mu(e, f)$, i.e., the coefficient of $f$ in $\sum_{e \in E^{\prime}} \alpha_{e} B_{T}(e)$ is well-defined.

The main theorem of this section is the following. In particular, this shows that $B_{w}$ is independent of $T$, which is not at all obvious from the definition. Also not obvious from the definition, at least in the case $\mathcal{C}$ consists of absolutely convergent series, is that $B_{w} \subseteq B_{t}$. It would be of interest to define $B_{w}$ without reference to a spanning tree.

Theorem 4.6 Let $(X, E)$ be a connected, compact, weakly Hausdorff edge space. Then $Z_{t}^{\perp}=B_{w}, B_{w}^{\perp}=Z_{t}$ and $B_{f} \subseteq B_{w}$, and, if $(X, E)$ is a compactification of a locally finite graph, then $B_{w} \subseteq B_{t}$. 
Proof: Let $T$ be a spanning tree of $(X, E)$. We begin by showing that if $b \in B_{w}$ and $z \in Z_{t}$, then $b \circ z=0$.

First of all, Theorem 2.2 implies

$$
b=\sum_{e \in E^{\prime}} \alpha_{e} B_{T}(e)=\sum_{e \in E^{\prime}} \alpha_{e} e+\sum_{f \in S}\left(\sum_{e \in E^{\prime}} \alpha_{e} \mu(e, f)\right) f,
$$

for $S=\bigcup_{e \in E^{\prime}}\left[\xi\left(B_{T}(e)\right) \backslash\{e\}\right]$.

On the other hand, Theorem 3.2 and Lemma 2.3 show that, for some $\gamma_{e}$,

$$
z=\sum_{e \notin T} \beta_{e} C_{T}(e)=\sum_{f \in S} \beta_{f} f+\sum_{e \in E^{\prime}}\left(\sum_{f \notin T} \beta_{f} \nu(e, f)\right) e+\sum_{e \notin\left(S \cup E^{\prime}\right)} \gamma_{e} e
$$

where $\nu(e, f)=0$ if $e \notin \xi\left(C_{T}(f)\right), \nu(e, f)=1$ if $e$ and $f$ have the same direction in $C_{T}(f)$, and $\nu(e, f)=-1$ if $e$ and $f$ have different directions in $C_{T}(f)$. We note that $\nu(e, f)=-\mu(e, f)$.

Obviously,

$$
b \circ z=\sum_{e \in E^{\prime}} \alpha_{e}\left(\sum_{f \in S} \beta_{f} \nu(e, f)\right)+\sum_{f \in S} \beta_{f}\left(\sum_{e \in E^{\prime}} \alpha_{e} \mu(e, f)\right) .
$$

The first of the two sums has terms of the form $\alpha_{e} \sum_{f \in S} \beta_{f} \nu(e, f)$. For each $f \in S$, the sum $\sum_{e \in E^{\prime}} \alpha_{e} \nu(e, f)=\sum_{e \in F} \alpha_{e} \nu(e, f)$ is in $\mathcal{C}$ (because $\left.\sum_{e \in F} \alpha_{e} \in \mathcal{C}\right)$, and, since $|S|<\infty, \sum_{f \in S} \beta_{f}\left(\sum_{e \in E^{\prime}} \alpha_{e} \nu(e, f)\right)$ is in $\mathcal{C}$ and is equal to $\sum_{e \in E^{\prime}} \alpha_{e}\left(\sum_{f \in S} \beta_{f} \nu(e, f)\right)$, so the latter is in $\mathcal{C}$, as required. The second of the two sums in $b \circ z$ has finitely many terms, each of which is of the form $\beta_{f} \sum_{e \in E^{\prime}} \alpha_{e} \mu(e, f)$, which is in $\mathcal{C}$ by the assumption $b \in B_{w}$. From these remarks, the first and second sums are both in $\mathcal{C}$ and their sum is 0 (recall $\nu(e, f)=-\mu(e, f)$ ), as required.

We have shown $B_{w} \subseteq \mathcal{Z}_{t}^{\perp}$ and $\mathcal{Z}_{t} \subseteq B_{w}^{\perp}$. Next we show $Z_{t}^{\perp} \subseteq B_{w}$; to this end, let $b=\sum_{e \in E} \alpha_{e} e \in Z_{t}^{\perp}$. By way of contradiction, suppose that

$$
\bigcup_{e \in T \cap \xi(b)} \xi\left(B_{T}(e)\right) \backslash\{e\}
$$

is infinite. We shall recursively select edges $e_{1}, e_{2}, \ldots$ and $f_{1}, f_{2}, \ldots$ If we have selected $e_{1}, \ldots, e_{i-1}$ and $f_{1}, \ldots, f_{i-1}$, then pick $e_{i} \in T \cap \xi(b)$ so that 
$B_{T}\left(e_{i}\right)$ has an edge $f_{i}$ not in $T \cup \bigcup_{j<i} B_{T}\left(e_{j}\right)$. By Theorem 2.2 each $B_{T}\left(e_{j}\right)$ is finite, so our assumption shows this is possible. Define

$$
\beta_{i}=\nu\left(e_{i}, f_{i}\right)\left(\alpha_{e_{i}}^{-1}-\sum_{j<i ; f_{j} \in B_{T}\left(e_{i}\right)} \beta_{j} \nu\left(e_{i}, f_{j}\right)\right) .
$$

Now set $z=\sum_{i=1}^{\infty} \beta_{i} C_{T}\left(f_{i}\right)$. Since this is a sum of fundamental cycles, Theorem 2.4 implies this is in $\mathcal{Z}_{t}$. Because $b \in \mathcal{Z}_{t}^{\perp}$, we should have $b \circ z=0$. However, the coefficient of $e_{i}$ in $z$ is $\sum_{j=1}^{\infty} \beta_{j} \nu\left(e_{i}, f_{j}\right)$, which is a finite sum, since the $C_{T}\left(f_{i}\right)$ make a thin family. Thus, in $b \circ z$ we find, for each $e_{i}$, the term

$$
\begin{aligned}
\alpha_{e_{i}} \sum_{j=1}^{\infty} \beta_{j} \nu\left(e_{i}, f_{j}\right) & =\alpha_{e_{i}} \beta_{i} \nu\left(e_{i}, f_{i}\right)+\alpha_{e_{i}} \sum_{j \neq i} \beta_{j} \nu\left(e_{i}, f_{j}\right) \\
& =1-\alpha_{e_{i}} \sum_{j<i} \beta_{j} \nu\left(e_{i}, f_{j}\right)+\alpha_{e_{i}} \sum_{j \neq i} \beta_{j} \nu\left(e_{i}, f_{j}\right) \\
& =1-\alpha_{e_{i}} \sum_{j>i} \beta_{j} \nu\left(e_{i}, f_{j}\right) .
\end{aligned}
$$

However, if $j>i$, then $f_{j}$ is not in $\xi\left(B_{T}\left(e_{i}\right)\right)$, so $\nu\left(e_{i}, f_{j}\right)=0$. Thus, for each $e_{i}, z \circ b$ has a 1 in the sum, so the sum is not in $\mathcal{C}$. Therefore,

$$
\bigcup_{e \in T \cap \xi(b)} \xi\left(B_{T}(e)\right) \backslash\{e\}
$$

must be finite.

Set $E^{\prime}=T \cap \xi(b)$ and $F=\left\{e \in T \mid \xi\left(B_{T}(e)\right) \neq\{e\}\right\}$. We would like to show that $\sum_{e \in F} \alpha_{e}$ is in $\mathcal{C}$. For each $f \in \cup_{e \in E^{\prime}} \xi\left(B_{T}(e)\right) \backslash\{e\}$ (of which there are only finitely many), observe that $b \circ C_{T}(f)=0$, so that $\sum_{e \in E^{\prime}} \alpha_{e} \mu(e, f)$ is in $\mathcal{C}$. But this implies $\sum_{e \in E^{\prime}, f \in \xi\left(B_{T}(e)\right)} \alpha_{e}$ is in $\mathcal{C}$. Now let $\bigcup_{e \in E^{\prime}} \xi\left(B_{T}(e)\right) \backslash\{e\}$ be $\left\{e_{1}, e_{2}, \ldots, e_{k}\right\}$ and, for $i=1,2, \ldots, k$, let $E_{i}^{\prime}=\left\{e \in E^{\prime} \mid e_{i} \in \xi\left(B_{T}(e)\right)\right\}$. Then each of the sums, for $1 \leq i \leq k, \sum_{e \in E_{i} \backslash \cup_{j<i} E_{j}} \alpha_{e}$ is in $\mathcal{C}$. Since there are finitely many of them, this implies $\sum_{e \in E^{\prime}} \alpha_{e}$ is in $\mathcal{C}$.

The preceding two paragraphs show $b^{\prime}=\sum_{e \in E^{\prime}} \alpha_{e} B_{T}(e) \in B_{w}$. Thus, $b-b^{\prime} \in \mathcal{Z}_{t}^{\perp}$. Since $\xi\left(b-b^{\prime}\right) \subseteq E \backslash T$, and, for every $f \notin T,\left(b-b^{\prime}\right) \circ C_{T}(f)=0$, we see that $b-b^{\prime}=0$, i.e., $b=b^{\prime} \in B_{w}$, as required. Hence $\mathcal{Z}_{t}^{\perp}=B_{w}$. Furthermore, since $\mathcal{Z}_{t}$ is generated by the thin family of fundamental cycles, Theorem 4.3 implies $\left(\mathcal{Z}_{t}^{\perp}\right)^{\perp}=\mathcal{Z}_{t}$, whence $B_{w}^{\perp}=\mathcal{Z}_{t}$. 
Finally, $B_{f} \subseteq\left(B_{f}^{\perp}\right)^{\perp}=\mathcal{Z}_{t}^{\perp}=B_{w}$ and, when $(X, E)$ is a compactification of a locally finite graph, $B_{w}=\mathcal{Z}_{t}^{\perp} \subseteq \mathcal{Z}_{f}^{\perp}=B_{t}$.

\section{An edge tripartition theorem}

To us, the most natural analogue of the Rosenstiehl-Read Edge Tripartition Theorem [6] would be the following.

Conjecture 5.1 Let $(X, E)$ be a compact, weakly Hausdorff edge space. Then, for every $e \in E$, exactly one of the following holds:

1. there is $a b \in B_{w} \cap \mathcal{Z}_{t}$ with $e \circ b=1$;

2. there is $a b \in B_{w}$ such that $e \circ b=1$ and $b-e \in \mathcal{Z}_{t}$; and

3. there is a $z \in \mathcal{Z}_{t}$ such that $e \circ z=1$ and $z-e \in B_{w}$.

We were not able to prove this, but were able to prove the following analogue.

Theorem 5.2 Let $(X, E)$ be a compactification of a locally finite graph. Then, for every $e \in E$, at least one of the following holds:

1. there is $a b \in B_{f} \cap \mathcal{Z}_{f}$ with $e \circ b=1$;

2. there is $a b \in B_{t}$ such that $e \circ b=1$ and $b-e \in \mathcal{Z}_{t}$; and

3. there is a $z \in \mathcal{Z}_{t}$ such that $e \circ z=1$ and $z-e \in B_{t}$.

If 1 holds, then neither (2) nor (3) holds.

As stated this result almost generalizes the Rosenstiehl-Read theorem, which asserts that, for finite graphs, EXACTLY one of the three holds (in that case, $\mathcal{Z}_{f}=\mathcal{Z}_{t}$ and $B_{f}=B_{t}$ ). Since it can happen that both (2) and (3) hold simultaneously, our theorem does not have this feature. This is essentially because we require $B_{t}$ rather than $B_{w}=Z_{t}^{\perp}$.

Proof of Theorem 5.2: Note that $B_{t}+\mathcal{Z}_{t}$ has a thin generating set, namely the union of the separate thin generating sets. Therefore, Theorem $4.3 \mathrm{im}-$ plies $\left(\left(B_{t}+\mathcal{Z}_{t}\right)^{\perp}\right)^{\perp}=B_{t}+\mathcal{Z}_{t}$. But Lemma 4.1 implies $\left(B_{t}+\mathcal{Z}_{t}\right)^{\perp}=B_{t}^{\perp} \cap \mathcal{Z}_{t}^{\perp}$. 
Theorem 4.5 implies $B_{t}^{\perp}=\mathcal{Z}_{f}$, while Theorem 4.6 implies $\mathcal{Z}_{t}^{\perp}=B_{w}$. Since $B_{w} \cap \mathcal{Z}_{f}=B_{f} \cap Z_{f}$, the result follows.

In a personal communication, Henning Bruhn informs us that he has proved the following.

Theorem 5.3 Let $(X, E)$ be a compactification of a locally finite graph. Then, for every $e \in E$, exactly one of the following holds:

1. there is $a b \in B_{t} \cap \mathcal{Z}_{t}$ with $e \circ b=1$;

2. there is $a b \in B_{f}$ such that $e \circ b=1$ and $b-e \in \mathcal{Z}_{f}$; and

3. there is a $z \in \mathcal{Z}_{f}$ such that $e \circ z=1$ and $z-e \in B_{f}$.

This form is different from ours; it regains the "EXACTLY" one feature of the Rosenstiehl-Read theorem, but it is unsatisfying in another way (which is also unsatisfying about our version): it requires more than one version of each of the cycle and bond spaces.

\section{Cycle spaces in different edge spaces}

Let $C_{1}$ and $C_{2}$ be compactifications of a topological space $X$. Then $C_{2} \leq C_{1}$ if there is a continuous function $f: C_{1} \rightarrow C_{2}$, with $f$ being the identity on $X$. It is well-known that, with this relation, the compactifications of a Hausdorff space make a lattice [5]. For Hausdorff spaces, such an $f$ is necessarily surjective. This is not generally true for weakly Hausdorff spaces, so some adjustment will be necessary for compactifications of edge spaces, such as assuming the compactifications are also edge spaces. The following simple theorem shows that compactifications related in this way have corresponding relations on their cycle spaces.

Theorem 6.1 Let $(X, E)$ and $(Y, F)$ be compact weakly Hausdorff edge spaces and let $h: X \rightarrow Y$ be a continuous function so that $h: E \rightarrow F$ is a bijection, $h(X \backslash E)=Y \backslash F$, and, if $e \in E$ is directed from $u$ to $v$, then $h(e)$ is directed from $h(u)$ to $h(v)$. Then, identifying each edge $e \in E$ with $h(e) \in F$ :

1. $B_{f}(Y, F) \subseteq B_{f}(X, E)$; 
2. $Z_{t}(X, E) \subseteq Z_{t}(Y, F)$;

3. if $T$ is a spanning tree of $Y$, then there is a spanning tree of $X$ containing $h^{-1}(T \cap F)$.

Proof: Let $b \in B_{f}(Y, F)$. Then $b=\sum_{i=1}^{n} \alpha_{i} \delta\left(A_{i}\right)$, for some partitions $\left(A_{1}, B_{1}\right), \ldots,\left(A_{n}, B_{n}\right)$ of $Y \backslash F$ into pairs of closed sets. For each $i=$ $1, \ldots, n,\left(h^{-1}\left(A_{i}\right), h^{-1}\left(B_{i}\right)\right)$ is a partition of $X \backslash E$ into closed sets. Clearly $h\left(\delta\left(h^{-1}\left(A_{i}\right)\right)\right)=\delta\left(A_{i}\right)$. Thus $b=\sum_{i=1}^{n} \alpha_{i} h\left(\delta\left(h^{-1}\left(A_{i}\right)\right)\right) \in B_{f}(X, E)$, as required for (1). Since $Z_{t}=B_{f}^{\perp}$ (Theorem 3.2), the fact that $A \subseteq B$ implies $B^{\perp} \subseteq A^{\perp}$ immediately yields (2).

Finally, if $T$ is a spanning tree of $(Y, F)$, then $T$ is closed in $Y$, so $h^{-1}(T)$ is closed in $X$. By [7, Thm. 3], there is a minimal connected subset $S$ of $X$ containing $h^{-1}(T)$. If $e \in S \cap E$ is such that $S-e$ is connected, then minimality of $S$ implies $e \in h^{-1}(T)$. Let $T_{1}$ and $T_{2}$ be the components of $T-e$; then $h^{-1}\left(T_{1}\right)$ and $h^{-1}\left(T_{2}\right)$ are non-empty and partition $S-e$ into closed sets. Thus, for $i=1,2, h^{-1}\left(T_{i}\right) \cup\{e\}$ is an open set in $S$, showing $S-e$ is not connected, a contradiction. Therefore, if $e \in S \cap E, S-e$ is not connected, i.e., $S$ is a spanning tree of $(X, E)$.

We hope in a further work to investigate the question of how to find a basis for $\mathcal{Z}(Y, F) / \mathcal{Z}(X, E)$. In [1], for a locally-finite graph embedded in the sphere with $k<\infty$ accumulation points, the closure of the embedded graph yields one edge space $(X, E)$ and this was compared with the Alexandroff compactification $(Y, E)$. In this special case, a basis for $\mathcal{Z}(Y, F) / \mathcal{Z}(X, E)$, with $\mathbb{F}=\mathbb{Z}_{2}$, was found.

\section{References}

[1] C.P. Bonnington and R.B. Richter, Graphs embedded in the plane with a bounded number of accumulation points, J. Graph Theory 44 (2003), no. $2,132-147$.

[2] R. Diestel, The cycle space of an infinite graph, Combin. Probab. Comput. 14 (2005), no. 1-2, 59-79.

[3] R. Diestel and D. Kühn, On infinite cycles. I, Combinatorica 24 (2004), no. 1, 69-89. 
[4] R. Diestel and D. Kühn, Topological paths, cycles and spanning trees in infinite graphs, European J. Combin. 25 (2004), no. 6, 835-862.

[5] R. Engelking, Outline of General Topology, North-Holland, Amsterdam, 1968.

[6] P. Rosenstiehl and R.C. Read, On the principal edge tripartition of a graph, Advances in graph theory (Cambridge Combinatorial Conf., Trinity College, Cambridge, 1977), Ann. Discrete Math. 3 (1978), 195-226.

[7] A. Vella and R.B. Richter, Cycle spaces in topological spaces, preprint.

[8] A. Vella, A Fundamentally Topological Perspective on Graph Theory, Ph.D. thesis, U. Waterloo, 2005. Available at http://etd.uwaterloo.ca/etd/avella2005.pdf

[9] S. Willard, General Topology, Addison-Wesley Publishing Co., Reading, Mass.-London-Don Mills, Ont. 1970. 